\title{
Temporal dynamics of microbial community structure and its effect on arsenic mobilization and transformation in Quaternary aquifers of the central Yangtze River Basin
}

\author{
T.L. Zheng ${ }^{1}$, Y. Deng ${ }^{1}$, H.C. Jiang ${ }^{2}$ \& Y.X. Wang ${ }^{2}$ \\ ${ }^{1}$ Geological Survey, China University of Geosciences, Wuhan, P.R. China \\ ${ }^{2}$ State Key Laboratory of Biogeology and Environmental Geology, \\ China University of Geosciences, Wuhan, P.R. China
}

\begin{abstract}
To elaborate the seasonal variation of groundwater arsenic (As) and its underlying regulating biogeochemical processes, long term biogeochemical monitoring and sediment incubation experiments were established in the aquifers from the Jianghan Plain, central Yangtze River basin. The results indicated that the groundwater As concentration exhibited significant seasonal variations (exceeding $800 \mu \mathrm{g} \mathrm{L}^{-1}$ ) between the pre-monsoon (January, February and March) and monsoon (June, July and August) season, which was regulated by the redox status fluctuation in the groundwater. The correlation between the $\mathrm{Fe}^{2+}$ and As concentrations in the groundwater from different seasons suggested the As mobilization in the aquifers was controlled by the iron mineral transformation. Furthermore, the incubation experiments with the sediments collected from different depths in the same aquifers indicated that the variation of microbial community was correlated with the released As concentration $(\mathrm{R}=0.7, \mathrm{P}<0.05)$ and the iron-reducing bacteria, including Pseudomonas, Clostridium and Geobacter, were the main drivers for the As mobilization from the sediments at $26 \mathrm{~m}$ and $36 \mathrm{~m}$ depth. In addition, the bacterial sulfate reducing process could affect the As temporal variation in groundwater through forming the Fe-sulfide minerals to scavenge the As in aqueous phase. Those results could provide new insights into the mechanism of As mobilization and seasonal As variation in groundwater systems.
\end{abstract}

\section{INTRODUCTION}

Natural elevation of arsenic (As) in groundwater poses serious health risk in various parts of the world. A prominent problem in the As-affected groundwater, which remains not well understood, is that the As concentrations vary temporally in groundwater. Recently, the shallow Quaternary aquifers in the Jianghan Plain, central Yangtze River Basin, has been reported with natural enrichment and seasonal variation of As in the groundwater, however little is known about the underlying regulating processes. Thus long term biogeochemical monitoring and anaerobic incubation experiments from aquifer sediments of different depths were conducted to reveal the underlying biogeochemical processes responsible for the As mobilization and temporal variation.

\section{METHODS AND MATERIALS}

\subsection{Study area}

Jianghan Plain is a semi-closed Quaternary basin with the subtropical monsoon climate, and it is formed by the alluvial sediments of the Yangtze River and its largest tributary Han River (Fig. 1). The Quaternary aquifer systems in the Jianghan Plain could be divided into two groups: 1) the Holocene unconfined aquifer, which is composed of Holocene alluvial and lacustrine

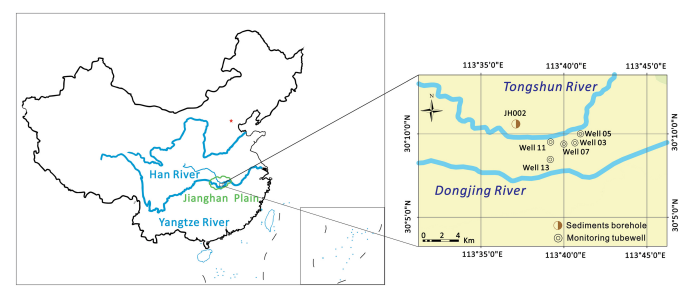

Figure 1. The geographical location of sampling borehole JH002 and monitoring wells 03, 05, 07, 11 and 13.

deposits (clay, silt and fine sand) in the depth of 3-15 m below the ground surface (bgs); 2) the late and middle Pleistocene confined aquifer, which is composed of alluvial sediments (medium coarse sand and gravel with clay lens) in the depth of $15-80 \mathrm{~m}$ bgs.

\subsection{Sampling and analysis}

The $\mathrm{pH}, \mathrm{Eh}, \mathrm{SES}$ and DO of groundwater were analyzed on site, and the groundwater samples of As, As(III)/As(V), cations, anions and total dissolved carbon were analyzed in laboratory. In addition, the sediment geochemistry characteristics were comprehensively analyzed by the X-ray Fluorescence Spectroscopy (XRF-1800, Shimadzu, Japan), X-Ray Diffractometer (D8-Focus, Germany) and the procedure of sequential extraction (Keon et al., 2001). 
The microbial community dynamics and variation of microbial functional genes were analyzed by the Illumina high-throughput sequencing and quantitative PCR techniques. The details of sample collection and analysis were described in a recent study (Deng et al., 2018).

\section{RESULTS AND DISCUSSION}

\subsection{Seasonal variation of microbial community compositions responding to the changing groundwater level and As concentration}

The results indicated that the microbial community compositions exhibited significant seasonal variation between the monsoon season and pre-monsoon season, in which the $\alpha$-Proteobacteria decreased from pre-monsoon season to monsoon season and the $\gamma$-Proteobacteria increased from pre-monsoon season to monsoon season (Fig. 2). The correralation between the microbial compostion dynamics and groundwater level fluctuation indicated that the observed seasonal variation of microbial community may be significantly affected by the varying ground water level. This observation may be ascribed to the fact that the groundwater level fluctuation changed the redox status in the aquifers, and supsequently altered the anaerobic and aerobic metabolism in the microbial community.

Furthermore, the corresponding seasonal variations of $\mathrm{Fe}(\mathrm{II})$ and As concentrations were observed in the groundwater, suggesting that the As concentration variation in the aquifer is associated with the $\mathrm{Fe}$ mineral transformations. Meanwhile, it is notable that the positively correlative variations between Geobacter $\mathrm{sp}$. and $\mathrm{As} / \mathrm{Fe}$ (II) were observed in the groundwater, while negative correlation between the Sideroxydans sp. and $\mathrm{As} / \mathrm{Fe}(\mathrm{II})$ was also observed. Those results suggested that the microbial functional groups could facilitate the As transformation in aquifers through affecting the redox cycle of iron.

\subsection{Microbial functional groups accounting for the As transformation and temporal variation}

In order to further understand the underlying regulating biogeochemical processes, aquifer sediment incubation experiments were conducted with sediments from different depths (Deng et al., 2018). The results indicated that the As mobilization was significantly associated with the reductive dissolution of iron oxide minerals and the variation of microbial community was correlated with As mobilization $(\mathrm{R}=0.7$, $\mathrm{P}<0.05)$ in JH26 and JH36. The OTUs associated with FeRB, including Pseudomonas, Pedobacter, Paenibacillus, Cellulomonas, Clostridium, Thiobacillus and Geobacter, were predominant in the microbial community, indicating those FeRB were the main drivers for As mobilization from the aquifer sediments.

In addition, the detection of $d s r B$ gene and the elimination of As in the late cultural period indicated that the bacteria sulfate reduction could scavenge the dissolved As in the solution. This observation was in

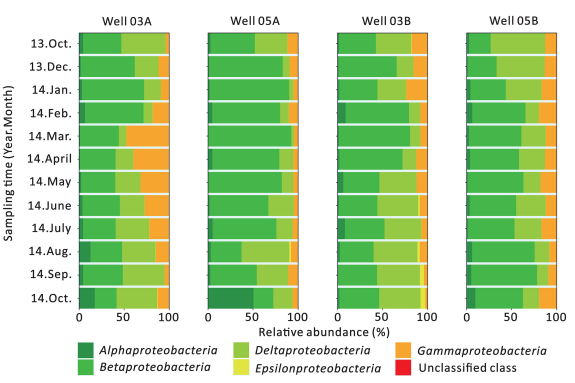

Figure 2. The seasonal variation of microbial community composition in the shallow groundwater from central Yangtze River Basin.
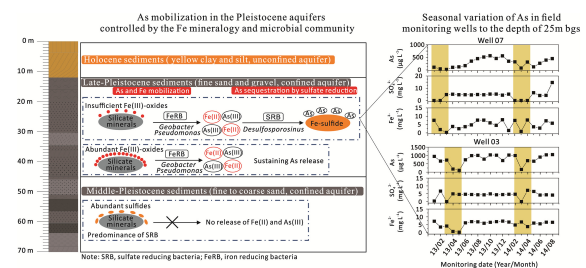

Figure 3. The generalization of As mobilization and temporal variation controlled by the Fe mineralogy and microbial community in the aquifer sediments from the Jianghan Plain, central Yangtze River Basin.

consistent with our field monitoring results in the premonsoon season, indicating that the microbial sulfate reduction process may also contribute to the temporal decrease of As concentration (Fig. 3).

\section{CONCLUSIONS}

The seasonal variation of As in aquifers could be affected by the variation of microbial community structures, and the As mobilization was mainly controlled by the distribution of microbial community and Fe mineralogy in the aquifer sediments.

\section{ACKNOWLEDGEMENTS}

The research was financially supported by the National Natural Science Foundation of China (Nos. 41572226 \& 41521001), Program of China Geological Survey (Nos. 121201001000150121), and State Key Laboratory of Biogeology and Environmental Geology, China University of Geosciences.

\section{REFERENCES}

Deng, Y., Zheng, T., Wang, Y., Liu, L., Jiang, H. \& Ma, T. 2018. Effect of microbially mediated iron mineral transformation on temporal variation of arsenic in the Pleistocene aquifers of the central Yangtze River basin. Sci. Total Environ. 619:1247-1258

Keon, N.E., Swartz, C.H., Brabander, D.J., Harvey, C. \& Hemond, H.F. 2001. Validation of an arsenic sequential extraction method for evaluating mobility in sediments. Environ. Sci. Technol. 35(13): 2778-2784. 